INPLASY

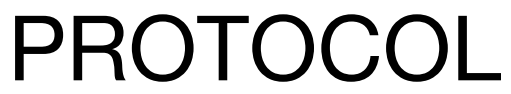

To cite: Zhang et al. Urbanrural differences in parenting style in China:a protocol for systematic review and meta analysis. Inplasy protocol 202050010. doi:

10.37766/inplasy2020.5.0010

Received: 03 May 2020

Published: 03 May 2020

Corresponding author: junhua zhang

junhuazh2003@163.com

Author Affiliation:

Nanjing Normal University

Support: Post-doctoral Fund of Jiangsu

Review Stage at time of this submission: The review has not yet started.

Conflicts of interest: No conflict of interest.

\section{Urban-rural differences in parenting style in China: a protocol for systematic review and meta analysis}

Zhang, J1; Zhang, Y2; Xu, F3.

Review question / Objective: Is there urban-rural difference in parenting style in China?

Condition being studied: Parenting style has profound influence on teenagers' self-concept and social development. Unearthing the specific origins of parenting is therefore a critically important research objective. Extant research on this topic has suggested that parenting behaviors are multidetermined and are associated with a wide range of contextual and familial characteristics. In China, there are great differences between urban and rural parents in terms of education level, number of children, lifestyle and customs, which will inevitably lead to differences in parenting styles between urban and rural areas. Many scholars have done a lot of investigation and have made many valuable discoveries. Some studies have found that rural fathers give their children more harsh punishment than city fathers, but there are also studies that hold the opposite view. Therefore, this study uses meta-analysis to conduct a more comprehensive evaluation of multiple independent studies and examine some factors that might result in differences among studies.

INPLASY registration number: This protocol was registered with the International Platform of Registered Systematic Review and Meta-Analysis Protocols (INPLASY) on 03 May 2020 and was last updated on 03 May 2020 (registration number INPLASY202050010).

\section{INTRODUCTION}

Review question / Objective: Is there urban-rural difference in parenting style in China?

Rationale: Existing literature shows several discrepancies in parenting style between urban and rural China, but conclusions are confusing. Therefore, the aim of this metaanalysis is to consider these inconsistencies and explore the influences of several moderator variables.

Condition being studied: Parenting style has profound influence on teenagers' selfconcept and social development. Unearthing the specific origins of parenting 
is therefore a critically important research objective. Extant research on this topic has suggested that parenting behaviors are multidetermined and are associated with a wide range of contextual and familial characteristics. In China, there are great differences between urban and rural parents in terms of education level, number of children, lifestyle and customs, which will inevitably lead to differences in parenting styles between urban and rural areas. Many scholars have done a lot of investigation and have made many valuable discoveries. Some studies have found that rural fathers give their children more harsh punishment than city fathers, but there are also studies that hold the opposite view. Therefore, this study uses meta-analysis to conduct a more comprehensive evaluation of multiple independent studies and examine some factors that might result in differences among studies.

\section{METHODS}

Search strategy: The following electronic databases will be searched with no language/date/type of document restrictions: PubMed, OVID, the Web of Knowledge, CNKI, Wan fang database and Chongqing VIP database. Search terms in PubMed are as follows:(China OR Chinese) AND (egna minnen av barn doms uppforstran OR embu OR parenting style OR parental rearing OR parenting behaviour OR parenting pattern OR education style OR My memories of upbringing) AND (urban OR city OR cities) AND (rural OR rural urban differences OR rural populations OR rural area OR countryside).

Participant or population: Parents in China.

Intervention: Living in rural areas in China.

Comparator: Parents living in urban areas in China.

Study designs to be included: Crosssection or observational studies.

Eligibility criteria: We will only includ studies that met the following criteria: (1) cross-sectional or observational study using Egna Minnen Beträffande Uppfostran( EMBU),Parental Bonding Instrument (PBI), and any other questionnaires including parenting style; (2) rural and urban group reported mean(M), standard deviation (SD), number (N) and other parameters such as $t, p$. The subjects are normal subjects and criminals and patients will be excluded.

Information sources: The following electronic databases will be searched with no language/date/type of document restrictions: PubMed, OVID, the Web of Knowledge, CNKI, Wan fang database and Chongqing VIP database.

Main outcome(s): The primary outcome will be score measured by the Chinese version of EMBU ,PBI and any other questionnaires including parenting style. EMBU is a Swedish self-report scale of perceptions about the behavior used by the respondent's parents in bringing them up. The Chinese version of the questionnaire was revised by Yue and comprises 66 items, with 58 in the six sub-scales about the father (F1 emotional warmth and understanding, F2 punishing, F3 overinterference, $\mathrm{F} 4$ favoritism, $\mathrm{F} 5$ rejection and F6 over-protection ) and 57 in the five subscales about the mother (M1; emotional warmth and understanding, M2 overinterference/over-protection, M3 rejection,M4 punishing and M5 favoritism). Responses are on a four-point Likert-type scale, from 1 (never) to 4 (always). Because of the one-child family planning policy adopted by China before 2011, most of the subjects in the study had no brothers or sisters. F4 and M5 are not suitable for the current situation, so no further investigation was made. The Chinese version of PBI included two dimensions of care and overprotection.

\section{Additional outcome(s): None.}

Quality assessment / Risk of bias analysis: Methodological quality of selected articles will be assessed by using the revised checklist by Chen20. It consists of 10 items, including study design, sampling 
method, rural group information, education level/age of child participants, data collection methods, response rate, comparison group information, measurement, statistical procedure and data sufficiency. Two authors will independently assess the quality of each selected study and disagreements will be resolved by further discussion.

Strategy of data synthesis: The following data will be extracted: 1. Study details: study citation, year(s) of study or publication, region (city or province that can be divided into western, central and eastern regions according to the level of economic development); 2.Participants details, including number, gender distribution, mean and range of age, in both groups; 3.Outcomes: M, SD of total score and sub-scores in both groups and other parameters such as $t, p$.

Subgroup analysis: Based on the age of subjects (High school and below VS college and beyond),regions, and questionnaires, we will do subgroup meta-analysis.

Sensibility analysis: We will plan a metaregression analysis including parenting style score as outcomes and year of publication, region, age group and rating on the checklist of quality assessment as regressor.

Country(ies) involved: China.

Keywords: Parenting style, rural, urban,meta-analysis, systematic review.

Contributions of each author:

Author 1 - Junhua Zhang.

Author 2 - Yu Zhang.

Author 3 - Fang Xu. 